\title{
Vertex- Edge Dominating Sets and Vertex-Edge Domination Polynomials of Wheels
}

\author{
A. Vijayan and T. Nagarajan \\ Associate Professor, Department of Mathematics, Nesamony Memorial Christian College, Marthandam, Tamil \\ Nadu, India \\ Assistant Professor, Department of Mathematics, Sivanthi Aditanar College, Pillayarpuram, Nagercoil, Tamil \\ Nadu, India.
}

Abstract: Let $G=(V, E)$ be a simple Graph. A set $S \subseteq V(G)$ is a vertex-edge dominating set (or simply $v e$-dominating set) if for all edges $e \in E(G)$, there exists a vertex $v \in S$ such that $v$ dominates $e$. In this paper, we study the concept of vertex-edge domination polynomial of wheels, $W_{n}$. The vertex-edge domination polynomial of $W_{n}$ is $D_{v e}\left(W_{n}, x\right)=\sum_{\mathrm{i}=1}^{\mathrm{n}} \mathrm{d}_{\mathrm{ve}}\left(\mathrm{W}_{\mathrm{n}}, \mathrm{i}\right) x^{\mathrm{i}}$, where $d_{v e}\left(W_{n}, i\right)$ is the number of vertex-edge dominating sets of $W_{n}$ with cardinality $i$. We obtain some properties of $D_{v e}\left(W_{n}, x\right)$ and its co-efficients. Also, we calculate the recursive formula to derive the vertex-edge domination polynomials of wheels.

Keywords: Star, wheel, vertex-edge dominating sets, vertex-edge domination number $\gamma_{v e}\left(W_{n}\right)$, vertex-edge domination polynomial

\section{Introduction}

Let $\mathrm{G}=(\mathrm{V}, \mathrm{E})$ be a simple graph of order $|\mathrm{V}|=\mathrm{n}$. A set $\mathrm{S} \subseteq \mathrm{V}$ is a dominating set of $\mathrm{G}$, if every vertex in VIS is adjacent to atleast one vertex in S. The domination number of a graph, denoted by $\gamma(\mathrm{G})$, is the minimum cardinality of the dominating sets in G. A set of vertices in a Graph G is said to be a vertex-edge dominating set, if for all edges $e \in E(G)$, there exists a vertex $v \in S$ such that $v$ dominates e. Otherwise, for a graph $\mathrm{G}=(\mathrm{V}, \mathrm{E})$, a vertex $\mathrm{u} \in \mathrm{V}(\mathrm{G})$ ve-dominates an edge $\mathrm{vw} \in \mathrm{E}(\mathrm{G})$ if (i) $\mathrm{u}=\mathrm{v}$ or $\mathrm{u}=\mathrm{w}$ (u is incident to $\mathrm{vw}$ ) or (ii) uv or uw is an edge in $\mathrm{G}$ ( $\mathrm{u}$ is incident to an edge is adjacent to vw).

The minimum cardinality of a ve-dominating set of $\mathrm{G}$ is called the vertex-edge domination number of $\mathrm{G}$, and is denoted by $\gamma_{\mathrm{ve}}(\mathrm{G})$. Let $\mathrm{W}_{\mathrm{n}}$ be the wheel with $\mathrm{n}$ vertices. In the next section, we construct the families of the vertex-edge dominating sets of wheels by recursive method. In section 3 , we use the results obtained in section 2 to study the vertex-edge domination polynomial of wheels.

We denote the set $\{1,2, \ldots, n\}$ by $[n]$, throughout this paper.

\section{Vertex-edge dominating sets of wheels}

Let $\mathrm{W}_{\mathrm{n}}, \mathrm{n} \geq 3$ be the wheel with $\mathrm{n}$ vertices $\mathrm{V}\left(\mathrm{W}_{\mathrm{n}}\right)=[\mathrm{n}]$ and $\mathrm{E}\left(\mathrm{W}_{\mathrm{n}}\right)=\{(1,2),(1,3), \ldots,(1, \mathrm{n})$, $(2,3),(3,4), \ldots,(n-1, n),(n, 2)\}$. Let $d_{v e}\left(W_{n}, i\right)$ be the family of vertex-edge dominating sets of $\mathrm{W}_{n}$ with cardinality i.

Lemma 2.1

The following results hold for all Graph $G$ with $|V(G)|=n$ vertices.

(ii) $\quad \mathrm{d}_{\mathrm{ve}}(\mathrm{G}, \mathrm{n}-1)=\mathrm{n}$

(iii) $\quad \mathrm{d}_{\mathrm{ve}}(\mathrm{G}, \mathrm{i})=0$ if $\mathrm{i}>\mathrm{n}$

(iv) $\quad \mathrm{d}_{\mathrm{ve}}(\mathrm{G}, 0)=0$

Lemma 2.2 [3]

For every $n \geq 5, j \geq\left\lceil\frac{n}{4}\right\rceil$

$d_{v e}\left(C_{n}, j\right)=d_{v e}\left(C_{n-1}, j-1\right)+d_{v e}\left(C_{n-2}, j-1\right)+d_{v e}\left(C_{n-3}, j-1\right)+d_{v e}\left(C_{n-4}, j-1\right)$

Theorem 2.3 [4]

Let $S_{n}, n \geq 3$ be a star Graph, then

$$
d_{v e}\left(S_{n}, i\right)=\left(\begin{array}{l}
n \\
i
\end{array}\right), \text { if } \mathrm{i} \leq \mathrm{n}
$$


(ii)

$$
d_{v e}\left(S_{n}, i\right)=\left\{\begin{array}{l}
d_{v e}\left(S_{n-1}, i\right)+1, \text { if } i=1 \\
d_{v e}\left(S_{n-1}, i\right)+d_{v e}\left(S_{n-1}, i-1\right), \text { if } 1<i \leq n
\end{array}\right.
$$

\section{Theorem 2.4}

Let $W_{n}, n \geq 4$ be the wheel Graph, then $d_{v e}\left(W_{n}, i\right)=d_{v e}\left(S_{n}, i\right)+d_{v e}\left(C_{n-1}, i\right)-\left(\begin{array}{c}n-1 \\ i\end{array}\right), i<n-1$

\section{Proof :}

Let $S_{n}$ be a star and $v \in v\left(S_{n}\right)$ such that $v$ is the center of $S_{n}$. Let $S_{n}$ be a spanning sub graph of $\mathrm{W}_{\mathrm{n}}$ and since $\mathrm{W}_{\mathrm{n}}-\mathrm{v}=\mathrm{C}_{\mathrm{n}-1}$ and $\mathrm{S}_{\mathrm{n}} \cup \mathrm{C}_{\mathrm{n}-1}=\mathrm{W}_{\mathrm{n}}$. The number of vertex-edge dominating sets of the wheel $\mathrm{w}_{\mathrm{n}}$ is the sum of the number of vertex edge dominating sets of the star $\left(\mathrm{S}_{\mathrm{n}}\right)$ and the number of vertexedge dominating sets of the cycle $\mathrm{C}_{n-1}$, and each time there are $\left(\begin{array}{c}n-1 \\ i\end{array}\right)$ sets of cardinality $i$ are not vertex-edge dominating sets.

Hence, $d_{v e}\left(W_{n}, i\right) \quad=\quad d_{v e}\left(S_{n}, i\right)+d_{v e}\left(C_{n-1}, i\right)-\left(\begin{array}{c}n-1 \\ i\end{array}\right), i<n-1$.

Theorem 2.5

Let $\mathrm{W}_{\mathrm{n}}, \mathrm{n} \geq 4$ be a wheel Graph, then

$$
d_{v e}\left(W_{n}, i\right)=d_{v e}\left(w_{n-1}, i-1\right)+d_{v e}\left(w_{n-2}, i-1\right)+d_{v e}\left(w_{n-3}, i-1\right)+d_{v e}\left(w_{n-4}, i-1\right)+\left(\begin{array}{l}
n-5 \\
i-1
\end{array}\right) .
$$

Proof:

Let $\mathrm{W}_{\mathrm{n}}, \mathrm{n} \geq 4$ be the wheel Graph. Then by theorem 2.3

$\mathrm{d}_{\mathrm{ve}}\left(\mathrm{S}_{\mathrm{n}}, \mathrm{i}\right)=\mathrm{d}_{\mathrm{ve}}\left(\mathrm{S}_{\mathrm{n}-1}, \mathrm{i}\right)+\mathrm{d}_{\mathrm{ve}}\left(\mathrm{S}_{\mathrm{n}-1}, \mathrm{i}-1\right)$

$$
\begin{array}{ll}
= & d_{v e}\left(S_{n-2}, i\right)+d_{v e}\left(S_{n-2}, i-1\right)+d_{v e}\left(S_{n-1}, i-1\right) \\
= & d_{v e}\left(S_{n-3}, i\right)+d_{v e}\left(S_{n-3}, i-1\right)+d_{v e}\left(S_{n-2}, i-1\right)+d_{v e}\left(S_{n-1}, i-1\right) \\
= & d_{v e}\left(S_{n-4}, i\right)+d_{v e}\left(S_{n-4}, i-1\right)+d_{v e}\left(S_{n-3}, i-1\right)+d_{v e}\left(S_{n-2}, i-1\right)+
\end{array}
$$

$\mathrm{d}_{\mathrm{ve}}\left(\mathrm{S}_{\mathrm{n}-1}, \mathrm{i}-1\right)$

by Theorem 2.3, $\mathrm{d}_{\mathrm{ve}}\left(\mathrm{S}_{\mathrm{n}-4}, \mathrm{i}\right)=\left(\begin{array}{c}\mathrm{n}-4 \\ \mathrm{i}\end{array}\right)$

Also, by Theorem 2.2,

$\mathrm{d}_{\mathrm{ve}}\left(\mathrm{C}_{\mathrm{n}}, \mathrm{i}\right)=\mathrm{d}_{\mathrm{ve}}\left(\mathrm{C}_{\mathrm{n}-1}, \mathrm{i}-1\right)+\mathrm{d}_{\mathrm{ve}}\left(\mathrm{C}_{\mathrm{n}-2}, \mathrm{i}-1\right)+\mathrm{d}_{\mathrm{ve}}\left(\mathrm{C}_{\mathrm{n}-3}, \mathrm{i}-1\right)+\mathrm{d}_{\mathrm{ve}}\left(\mathrm{C}_{\mathrm{n}-4}, \mathrm{i}-1\right)$

by Theorem 2.4 ,

$$
\begin{aligned}
& \mathrm{d}_{\mathrm{ve}}\left(\mathrm{W}_{\mathrm{n}}, \mathrm{i}\right)=\mathrm{d}_{\mathrm{ve}}\left(\mathrm{S}_{\mathrm{n}}, \mathrm{i}\right)+\mathrm{d}_{\mathrm{ve}}\left(\mathrm{C}_{\mathrm{n}-1}, \mathrm{i}\right)-\left(\begin{array}{c}
\mathrm{n}-1 \\
\mathrm{i}
\end{array}\right) \\
& =\quad d_{\mathrm{ve}}\left(\mathrm{S}_{\mathrm{n}-4}, \mathrm{i}\right)+\mathrm{d}_{\mathrm{ve}}\left(\mathrm{S}_{\mathrm{n}-4}, \mathrm{i}-1\right)+\mathrm{d}_{\mathrm{ve}}\left(\mathrm{S}_{\mathrm{n}-3}, \mathrm{i}-1\right)+\mathrm{d}_{\mathrm{ve}}\left(\mathrm{S}_{\mathrm{n}-2}\right. \text {, } \\
& \mathrm{i}-1)+\mathrm{d}_{\mathrm{ve}}\left(\mathrm{S}_{\mathrm{n}-1}, \mathrm{i}-1\right)+\mathrm{d}_{\mathrm{ve}}\left(\mathrm{C}_{\mathrm{n}-2}, \mathrm{i}-1\right)+\mathrm{d}_{\mathrm{ve}}\left(\mathrm{C}_{\mathrm{n}-3}, \mathrm{i}-1\right)+\mathrm{d}_{\mathrm{ve}}\left(\mathrm{C}_{\mathrm{n}-4}, \mathrm{i}-1\right)+ \\
& \mathrm{d}_{\mathrm{ve}}\left(\mathrm{C}_{\mathrm{n}-5}, \mathrm{i}-1\right)-\left(\begin{array}{c}
\mathrm{n}-1 \\
\mathrm{i}
\end{array}\right) \\
& \begin{array}{l}
=\quad \mathrm{d}_{\mathrm{ve}}\left(\mathrm{S}_{\mathrm{n}-1}, \mathrm{i}-1\right)+\mathrm{d}_{\mathrm{ve}}\left(\mathrm{C}_{\mathrm{n}-2}, \mathrm{i}-1\right)-\left(\begin{array}{c}
\mathrm{n}-2 \\
\mathrm{i}-1
\end{array}\right) \\
+\quad \mathrm{d}_{\mathrm{ve}}\left(\mathrm{S}_{\mathrm{n}-2}, \mathrm{i}-1\right)+\mathrm{d}_{\mathrm{ve}}\left(\mathrm{C}_{\mathrm{n}-3}, \mathrm{i}-1\right)-\left(\begin{array}{c}
\mathrm{n}-3 \\
\mathrm{i}-1
\end{array}\right) \\
+\quad \mathrm{d}_{\mathrm{ve}}\left(\mathrm{S}_{\mathrm{n}-3}, \mathrm{i}-1\right)+\mathrm{d}_{\mathrm{ve}}\left(\mathrm{C}_{\mathrm{n}-4}, \mathrm{i}-1\right)-\left(\begin{array}{c}
\mathrm{n}-4 \\
\mathrm{i}-1
\end{array}\right)
\end{array}
\end{aligned}
$$




$$
\begin{aligned}
& +\quad \mathrm{d}_{\mathrm{ve}}\left(\mathrm{S}_{\mathrm{n}-4}, \mathrm{i}-1\right)+\mathrm{d}_{\mathrm{ve}}\left(\mathrm{C}_{\mathrm{n}-5}, \mathrm{i}-1\right)-\left(\begin{array}{c}
\mathrm{n}-5 \\
\mathrm{i}-1
\end{array}\right) \\
& +\quad\left(\begin{array}{c}
n-2 \\
i-1
\end{array}\right)+\left(\begin{array}{l}
n-3 \\
i-1
\end{array}\right)+\left(\begin{array}{c}
n-4 \\
i-1
\end{array}\right)+\left(\begin{array}{c}
n-5 \\
i-1
\end{array}\right) \\
& +\left(\begin{array}{c}
n-4 \\
\mathrm{i}
\end{array}\right)-\left(\begin{array}{c}
\mathrm{n}-1 \\
\mathrm{i}
\end{array}\right) \\
& \mathrm{d}_{\mathrm{ve}}\left(\mathrm{W}_{\mathrm{n}}, \mathrm{i}\right)=\mathrm{d}_{\mathrm{ve}}\left(\mathrm{W}_{\mathrm{n}-1}, \mathrm{i}-1\right)+\mathrm{d}_{\mathrm{ve}}\left(\mathrm{W}_{\mathrm{n}-2}, \mathrm{i}-1\right)+\mathrm{d}_{\mathrm{ve}}\left(\mathrm{W}_{\mathrm{n}-3}, \mathrm{i}-1\right)+\mathrm{d}_{\mathrm{ve}}\left(\mathrm{W}_{\mathrm{n}-4}, \mathrm{i}-1\right) \\
& +\left(\begin{array}{c}
n-2 \\
i-1
\end{array}\right)+\left(\begin{array}{c}
n-3 \\
i-1
\end{array}\right)+\left(\begin{array}{c}
n-4 \\
i-1
\end{array}\right)+\left(\begin{array}{c}
n-5 \\
i-1
\end{array}\right) \\
& +\left(\begin{array}{c}
n-4 \\
\mathrm{i}
\end{array}\right)-\left(\begin{array}{c}
\mathrm{n}-1 \\
\mathrm{i}
\end{array}\right) \\
& \text { Consider }\left(\begin{array}{c}
n-4 \\
i-1
\end{array}\right)+\left(\begin{array}{c}
n-4 \\
i
\end{array}\right) \\
& =\frac{(n-4) !}{(i-1) !(n-4-i+1) !}+\frac{(n-4) !}{i !(n-4-i) !} \\
& =\frac{(n-4) !}{(i-1) !(n-i-3) !}+\frac{(n-4) !}{i !(n-i-4) !} \\
& = \\
& \frac{(n-4) !}{(i-1) !(n-i-3) !}+\frac{(n-4) !(n-i-3)}{i \times(i-1) !(n-i-4) !(n-i-3)} \\
& = \\
& \frac{(n-4) !}{(i-1) !(n-i-3) !}+\frac{(n-4) !(n-i-3)}{i \times(i-1) !(n-i-3) !} \\
& =\frac{(n-4) !}{(i-1) !(n-i-3) !}\left[1+\frac{n-i-3}{i}\right] \\
& =\frac{(n-4) !}{(i-1) !(n-i-3) !}\left[\frac{i+n-i-3}{i}\right] \\
& =\frac{(n-4) !}{(i-1) !(n-i-3) !} \times \frac{n-3}{i} \\
& =\frac{(n-3) !}{i !(n-i-3) !}=\left(\begin{array}{c}
n-3 \\
i
\end{array}\right) \\
& \left(\begin{array}{c}
n-3 \\
i-1
\end{array}\right)+\left(\begin{array}{c}
n-3 \\
i
\end{array}\right)=\left(\begin{array}{c}
n-2 \\
i
\end{array}\right) \\
& \left(\begin{array}{c}
n-2 \\
i-1
\end{array}\right)+\left(\begin{array}{c}
n-2 \\
i
\end{array}\right)=\left(\begin{array}{c}
n-1 \\
i
\end{array}\right) \\
& \therefore \mathrm{d}_{\mathrm{ve}}\left(\mathrm{W}_{\mathrm{n}}, \mathrm{i}\right)=\mathrm{d}_{\mathrm{ve}}\left(\mathrm{W}_{\mathrm{n}-1}, \mathrm{i}-1\right)+\mathrm{d}_{\mathrm{ve}}\left(\mathrm{W}_{\mathrm{n}-2}, \mathrm{i}-1\right)+\mathrm{d}_{\mathrm{ve}}\left(\mathrm{W}_{\mathrm{n}-3}, \mathrm{i}-1\right)+\mathrm{d}_{\mathrm{ve}}\left(\mathrm{W}_{\mathrm{n}-4}, \mathrm{i}-1\right)
\end{aligned}
$$




$$
\begin{aligned}
& +\left(\begin{array}{c}
n-2 \\
i-1
\end{array}\right)+\left(\begin{array}{c}
n-3 \\
i-1
\end{array}\right)+ \\
& \left(\begin{array}{c}
n-4 \\
i-1
\end{array}\right)+\left(\begin{array}{c}
n-4 \\
i
\end{array}\right)+\left(\begin{array}{c}
n-5 \\
i-1
\end{array}\right)-\left(\begin{array}{c}
n-1 \\
i
\end{array}\right) \\
& =\quad \mathrm{d}_{\mathrm{ve}}\left(\mathrm{W}_{\mathrm{n}-1}, \mathrm{i}-1\right)+\mathrm{d}_{\mathrm{ve}}\left(\mathrm{W}_{\mathrm{n}-2}, \mathrm{i}-1\right)+\mathrm{d}_{\mathrm{ve}}\left(\mathrm{W}_{\mathrm{n}-3}, \mathrm{i}-1\right)+ \\
& \mathrm{d}_{\mathrm{ve}}\left(\mathrm{W}_{\mathrm{n}-4}, \mathrm{i}-1\right) \\
& \left(\begin{array}{c}
n-3 \\
i
\end{array}\right)+\left(\begin{array}{c}
n-5 \\
i-1
\end{array}\right)-\left(\begin{array}{c}
n-1 \\
i
\end{array}\right) \\
& =\quad d_{v e}\left(W_{n-1}, i-1\right)+d_{v e}\left(W_{n-2}, i-1\right)+d_{v e}\left(W_{n-3}, i-1\right)+ \\
& \mathrm{d}_{\mathrm{ve}}\left(\mathrm{W}_{\mathrm{n}-4}, \mathrm{i}-1\right) \\
& \left(\begin{array}{c}
n-5 \\
i-1
\end{array}\right)-\left(\begin{array}{c}
n-1 \\
i
\end{array}\right) \\
& +\left(\begin{array}{c}
n-2 \\
i-1
\end{array}\right)+\left(\begin{array}{c}
n-3 \\
i-1
\end{array}\right)+ \\
& \mathrm{d}_{\mathrm{ve}}\left(\mathrm{W}_{\mathrm{n}-4}, \mathrm{i}-1\right) \\
& \left(\begin{array}{c}
n-1 \\
i
\end{array}\right) \\
& +\left(\begin{array}{c}
n-2 \\
i-1
\end{array}\right)+\left(\begin{array}{c}
n-2 \\
i
\end{array}\right)+ \\
& d_{v e}\left(W_{n-4}, i-1\right)+\left(\begin{array}{c}
n-5 \\
i-1
\end{array}\right) \\
& =\quad d_{v e}\left(W_{n-1}, i-1\right)+d_{v e}\left(W_{n-2}, i-1\right)+d_{v e}\left(W_{n-3}, i-1\right)+ \\
& +\quad\left(\begin{array}{c}
n-1 \\
i
\end{array}\right)+\left(\begin{array}{c}
n-5 \\
i-1
\end{array}\right)-
\end{aligned}
$$

\begin{tabular}{|c|c|c|c|c|c|c|c|c|c|c|c|c|c|c|c|}
\hline $\mathrm{n}$ & 1 & 2 & 3 & 4 & 5 & 6 & 7 & 8 & 9 & 10 & 11 & 12 & 13 & 14 & 15 \\
\hline 1 & 1 & & & & & & & & & & & & & & \\
\hline 2 & 2 & 1 & & & & & & & & & & & & & \\
\hline 3 & 3 & 3 & 1 & & & & & & & & & & & & \\
\hline 4 & 4 & 6 & 4 & 1 & & & & & & & & & & & \\
\hline 5 & 5 & 10 & 10 & 5 & 1 & & & & & & & & & & \\
\hline 6 & 1 & 15 & 20 & 15 & 6 & 1 & & & & & & & & & \\
\hline 7 & 1 & 15 & 35 & 35 & 21 & 7 & 1 & & & & & & & & \\
\hline 8 & 1 & 14 & 49 & 70 & 56 & 28 & 8 & 1 & & & & & & & \\
\hline 9 & 1 & 12 & 60 & 118 & 126 & 84 & 36 & 9 & 1 & & & & & & \\
\hline 10 & 1 & 9 & 66 & 174 & 243 & 210 & 120 & 45 & 10 & 1 & & & & & \\
\hline 11 & 1 & 5 & 65 & 230 & 412 & 452 & 330 & 165 & 55 & 11 & 1 & & & & \\
\hline 12 & 1 & 5 & 55 & 275 & 627 & 858 & 781 & 495 & 220 & 66 & 12 & 1 & & & \\
\hline 13 & 1 & 5 & 45 & 295 & 867 & 1464 & 1632 & 1275 & 715 & 286 & 78 & 13 & 1 & & \\
\hline 14 & 1 & 5 & 36 & 291 & 1092 & 2275 & 3068 & 2899 & 1989 & 1001 & 364 & 91 & 14 & 11 & \\
\hline & & 5 & & 267 & & & & & & & & & & & \\
\hline
\end{tabular}

Table 1: $d_{v e}\left(W_{n}, i\right)$, The Number of Vertex-Edge dominating sets of $W_{n}$ with cardinality $i$

\section{Theorem 2.6}

For every $\mathrm{n} \in \mathrm{Z}^{+}, \mathrm{n} \geq 4$

(i) $\mathrm{d}_{\mathrm{ve}}\left(\mathrm{W}_{\mathrm{n}}, 1\right)=1, \mathrm{n}>5$ 
(ii) $\mathrm{d}_{\mathrm{ve}}\left(\mathrm{W}_{\mathrm{n}}, 2\right)=\mathrm{n}-1, \mathrm{n}>9$

(iii) $\mathrm{d}_{\mathrm{ve}}\left(\mathrm{w}_{\mathrm{n}}, \mathrm{n}-2\right)=\left(\begin{array}{l}\mathrm{n} \\ 2\end{array}\right)$

(iv) $\gamma_{\mathrm{ve}}\left(\mathrm{W}_{\mathrm{n}}\right)=1$

(v) $\mathrm{d}_{\mathrm{ve}}\left(\mathrm{W}_{\mathrm{n}}, \mathrm{i}\right)=\mathrm{d}_{\mathrm{ve}}\left(\mathrm{W}_{\mathrm{n}-1}, \mathrm{i}-1\right)$

$$
\begin{aligned}
& +\mathrm{d}_{\mathrm{ve}}\left(\mathrm{W}_{\mathrm{n}-2}, \mathrm{i}-1\right) \\
& +\mathrm{d}_{\mathrm{ve}}\left(\mathrm{W}_{\mathrm{n}-3}, \mathrm{i}-1\right) \\
& +\mathrm{d}_{\mathrm{ve}}\left(\mathrm{W}_{\mathrm{n}-4}, \mathrm{i}-1\right), \mathrm{i} \geq \mathrm{n}-3, \mathrm{i} \neq \mathrm{n}-4
\end{aligned}
$$

(vi) $\mathrm{d}_{\mathrm{ve}}\left(\mathrm{W}_{\mathrm{n}}, \mathrm{i}\right)=\mathrm{d}_{\mathrm{ve}}\left(\mathrm{W}_{\mathrm{n}-1}, \mathrm{i}-1\right)+\mathrm{d}_{\mathrm{ve}}\left(\mathrm{W}_{\mathrm{n}-2}, \mathrm{i}-1\right)+\mathrm{d}_{\mathrm{ve}}\left(\mathrm{W}_{\mathrm{n}-3}, \mathrm{i}-1\right)+\mathrm{d}_{\mathrm{ve}}\left(\mathrm{W}_{\mathrm{n}-4}, \mathrm{i}-1\right)+1, \mathrm{i}=\mathrm{n}-4$ Proof:

(i) Let $\mathrm{W}_{\mathrm{n}}$ be the wheel and $\mathrm{v} \in \mathrm{V}\left(\mathrm{W}_{\mathrm{n}}\right)$ such that $\mathrm{v}$ is the center of $\mathrm{W}_{\mathrm{n}}$. then from table, $\mathrm{d}_{\mathrm{ve}}\left(\mathrm{W}_{\mathrm{n}}, 1\right)$ $=1, \mathrm{n}>5$

(ii) by Theorem 2.4, $\mathrm{d}_{\mathrm{ve}}\left(\mathrm{W}_{\mathrm{n}}, \mathrm{i}\right)=\mathrm{d}_{\mathrm{ve}}\left(\mathrm{S}_{\mathrm{n}}, \mathrm{i}\right)+\mathrm{d}_{\mathrm{ve}}\left(\mathrm{C}_{\mathrm{n}-1}, \mathrm{i}\right)-\left(\begin{array}{c}\mathrm{n}-1 \\ \mathrm{i}\end{array}\right)$

$$
\begin{aligned}
& \therefore \mathrm{d}_{\mathrm{ve}}\left(\mathrm{W}_{\mathrm{n}}, 2\right)=\mathrm{d}_{\mathrm{ve}}\left(\mathrm{S}_{\mathrm{n}}, 2\right)+\mathrm{d}_{\mathrm{ve}}\left(\mathrm{c}_{\mathrm{n}-1}, 2\right)-\left(\begin{array}{c}
\mathrm{n}-1 \\
2
\end{array}\right) \\
& =\left(\begin{array}{c}
\mathrm{n} \\
2
\end{array}\right)+0-\left(\begin{array}{c}
\mathrm{n}-1 \\
2
\end{array}\right) \quad \text { (by theorem 2.3) }
\end{aligned}
$$

$\left(\square \mathrm{d}_{\mathrm{ve}}\left(\mathrm{C}_{\mathrm{n}-1}, 2\right)=0, \mathrm{n}>9\right)$

$$
\begin{aligned}
& =\left(\begin{array}{l}
n \\
2
\end{array}\right)-\left(\begin{array}{c}
n-1 \\
2
\end{array}\right) \\
& =\frac{\mathrm{n} !}{2 !(\mathrm{n}-2) !}-\frac{(\mathrm{n}-1) !}{2 !(\mathrm{n}-3) !} \\
& =\frac{n !}{2 !(n-2) !}-\frac{n(n-1) !(n-2)}{n \times 2 ! x(n-3) !(n-2)} \\
& =\frac{n !}{2 !(n-2) !}-\frac{n !(n-2)}{n \times 2 ! x(n-2) !} \\
& =\frac{\mathrm{n} !}{2 !(\mathrm{n}-2) !}\left[1-\frac{\mathrm{n}-2}{\mathrm{n}}\right] \\
& =\frac{\mathrm{n} !}{2 !(\mathrm{n}-2) !}\left[\frac{\mathrm{n}-(\mathrm{n}-2)}{\mathrm{n}}\right] \\
& =\frac{\mathrm{n} !}{2 !(\mathrm{n}-2) !} \times \frac{2}{\mathrm{n}}=\frac{\mathrm{n}(\mathrm{n}-1)(\mathrm{n}-2) ! \times 2}{2 ! \times(\mathrm{n}-2) \times \mathrm{n}} \\
& =\mathrm{n}-1
\end{aligned}
$$

(iii) by theorem 2.4 ,

$$
\begin{aligned}
& d_{v e}\left(W_{n}, i\right)=d_{v e}\left(S_{n}, i\right)+d_{v e}\left(c_{n-1}, i\right)-\left(\begin{array}{c}
n-1 \\
i
\end{array}\right) \\
& \therefore \quad d_{v e}\left(W_{n}, n-2\right)=d_{v e}\left(S_{n}, n-2\right)+d_{v e}\left(C_{n-1}, n-2\right)-\left(\begin{array}{c}
n-1 \\
n-2
\end{array}\right)
\end{aligned}
$$




$$
\begin{aligned}
& =\left(\begin{array}{l}
\mathrm{n} \\
\mathrm{n}-2
\end{array}\right)+(\mathrm{n}-1)-\left(\begin{array}{c}
\mathrm{n}-1 \\
\mathrm{n}-2
\end{array}\right) \\
& =\frac{\mathrm{n} !}{(\mathrm{n}-2) ! 2 !}+(\mathrm{n}-1)-\frac{(\mathrm{n}-1) !}{(\mathrm{n}-2) ! \times 1} \\
& =\frac{\mathrm{n}(\mathrm{n}-1)(\mathrm{n}-2) !}{2 \times(\mathrm{n}-2) !}+(\mathrm{n}-1)-\frac{(\mathrm{n}-1)(\mathrm{n}-2) !}{1 ! \times(\mathrm{n}-2) !} \\
& =\frac{\mathrm{n}(\mathrm{n}-1)}{2}+(\mathrm{n}-1)-(\mathrm{n}-1) \\
& =\frac{\mathrm{n}(\mathrm{n}-1)}{2}=\left(\frac{\mathrm{n}}{2}\right)
\end{aligned}
$$

(iv) The center vertex $\mathrm{v}$ is enough to cover all the vertices and edges of $\mathrm{W}_{\mathrm{n}}$. therefore, the minimum cardinality of the vertex-edge dominating set of $\mathrm{W}_{\mathrm{n}}$ is 1

$\therefore \gamma_{\mathrm{ve}}\left(\mathrm{W}_{\mathrm{n}}\right)=1$

(v) By theorem 2.5,

$$
d_{v e}\left(W_{n}, i\right)=d_{v e}\left(W_{n-1}, i-1\right)+d_{v e}\left(W_{n-z}, i-1\right)+d_{v e}\left(W_{n-3}, i-1\right)+d_{v e}\left(W_{n-4}, i-1\right)+\left(\begin{array}{l}
n-5 \\
i-1
\end{array}\right)
$$

Since $\mathrm{i} \geq \mathrm{n}-3$,

$\therefore \mathrm{i}-1 \geq \mathrm{n}-4 \therefore \mathrm{i}-1=\mathrm{n}-4, \mathrm{n}-3, \mathrm{n}-2, \mathrm{n}-1, \mathrm{n}, \ldots$

then $\left(\begin{array}{c}n-5 \\ i-1\end{array}\right)=0$

and if $\mathrm{i}=\mathrm{n}-4 \therefore\left(\begin{array}{c}\mathrm{n}-5 \\ \mathrm{i}-1\end{array}\right) \neq 0 \therefore \mathrm{i} \neq \mathrm{n}-4$

substitute in (1) we get

$$
\begin{aligned}
\mathrm{d}_{\mathrm{ve}}\left(\mathrm{W}_{\mathrm{n}, \mathrm{i}} \mathrm{i}\right)=\mathrm{d}_{\mathrm{ve}}\left(\mathrm{W}_{\mathrm{n}-1}, \mathrm{i}-1\right)+\mathrm{d}_{\mathrm{ve}}\left(\mathrm{W}_{\mathrm{n}-2}, \mathrm{i}-1\right) & \\
& +\mathrm{d}_{\mathrm{ve}}\left(\mathrm{W}_{\mathrm{n}-3}, \mathrm{i}-1\right) \\
\quad & \quad \mathrm{d}_{\mathrm{ve}}\left(\mathrm{W}_{\mathrm{n}-4}, \mathrm{i}-1\right)+0 \\
= & \mathrm{d}_{\mathrm{ve}}\left(\mathrm{W}_{\mathrm{n}-1}, \mathrm{i}-1\right)+\mathrm{d}_{\mathrm{ve}}\left(\mathrm{W}_{\mathrm{n}-2}, \mathrm{i}-1\right) \\
\quad & \quad \mathrm{d}_{\mathrm{ve}}\left(\mathrm{W}_{\mathrm{n}-3}, \mathrm{i}-1\right)+\mathrm{d}_{\mathrm{ve}}\left(\mathrm{W}_{\mathrm{n}-4}, \mathrm{i}-1\right), \quad \mathrm{i} \geq \mathrm{n}-3, \mathrm{i} \neq \mathrm{n}-4
\end{aligned}
$$

(vi) by theorm 2.5 ,

$$
d_{\mathrm{ve}}\left(\mathrm{W}_{\mathrm{n}}, \mathrm{i}\right)=\mathrm{d}_{\mathrm{ve}}\left(\mathrm{W}_{\mathrm{n}-1}, \mathrm{i}-1\right)+\mathrm{d}_{\mathrm{ve}}\left(\mathrm{W}_{\mathrm{n}-2}, \mathrm{i}-1\right)
$$

$$
\begin{aligned}
& +d_{v e}\left(W_{n-3}, i-1\right) \\
& +d_{v e}\left(W_{n-4}, i-1\right)+\left(\begin{array}{l}
n-5 \\
i-1
\end{array}\right)
\end{aligned}
$$

$\mathrm{i}=\mathrm{n}-4 \therefore\left(\begin{array}{c}\mathrm{n}-5 \\ \mathrm{i}-1\end{array}\right)=\left(\begin{array}{l}\mathrm{n}-5 \\ \mathrm{n}-5\end{array}\right)=1$

$\therefore \mathrm{d}_{\mathrm{ve}}\left(\mathrm{W}_{\mathrm{n}, \mathrm{i}}\right)=\mathrm{d}_{\mathrm{ve}}\left(\mathrm{W}_{\mathrm{n}-1}, \mathrm{i}-1\right)+\mathrm{d}_{\mathrm{ve}}\left(\mathrm{W}_{\mathrm{n}-2}, \mathrm{i}-1\right)+\mathrm{d}_{\mathrm{ve}}\left(\mathrm{W}_{\mathrm{n}-3}, \mathrm{i}-1\right)+\mathrm{d}_{\mathrm{ve}}\left(\mathrm{W}_{\mathrm{n}-4}, \mathrm{i}-1\right)+1, \mathrm{i}=\mathrm{n}-4$.

\section{Definition 3.1}

\section{Vertx - edge domination polynomial of wheel}

Let $d_{v e}\left(W_{n}, i\right)$ be the family of vertex - edge dominating sets of a wheel $W_{n}$ with cardinality $i$, then vertex-edge domination polynomial of $\mathrm{W}_{\mathrm{n}}$ is defined as

Theorem 3.2

$$
\mathrm{D}_{\mathrm{ve}}\left(\mathrm{W}_{\mathrm{n}}, x\right)=\sum_{\mathrm{i}=1}^{\mathrm{n}} \mathrm{d}_{\mathrm{ve}}\left(\mathrm{W}_{\mathrm{n}}, \mathrm{i}\right) x^{\mathrm{i}}
$$


$\mathrm{D}_{\text {ve }}\left(\mathrm{W}_{\mathrm{n}}, x\right)$ is the vertex-edge domination polynomial of wheel $\mathrm{W}_{\mathrm{n}}, \mathrm{n} \geq 5$

(i) $\mathrm{D}_{\text {ve }}\left(\mathrm{W}_{\mathrm{n}}, x\right)=\mathrm{D}_{\text {ve }}\left(\mathrm{S}_{\mathrm{n}}, x\right)+\mathrm{D}_{\text {ve }}\left(\mathrm{C}_{\mathrm{n}-1}, x\right)-\left((1+x)^{\mathrm{n}-1}-1\right)$

(ii) $\mathrm{D}_{\text {ve }}\left(\mathrm{W}_{\mathrm{n}}, x\right)=x \mathrm{D}_{\text {ve }}\left(\mathrm{W}_{\mathrm{n}-1}, x\right)$

$$
\begin{aligned}
& +x \mathrm{D}_{\text {ve }}\left(\mathrm{W}_{\mathrm{n}-2}, x\right) \\
& +x \mathrm{D}_{\text {ve }}\left(\mathrm{W}_{\mathrm{n}-3}, x\right) \\
& +x \mathrm{D}_{\text {ve }}\left(\mathrm{W}_{\mathrm{n}-4}, x\right)+x(1+x)^{\mathrm{n}-5}
\end{aligned}
$$

Proof :

(i) From the definition of vertex-edge domination polynomial of wheel, we have

$$
\begin{aligned}
\mathrm{D}_{\mathrm{ve}}\left(\mathrm{W}_{\mathrm{n}, x}\right)=\sum_{\mathrm{i}=1}^{\mathrm{n}} \mathrm{d}_{\mathrm{ve}}\left(\mathrm{W}_{\mathrm{n}, \mathrm{i}}\right) x^{\mathrm{i}} & \\
& =\sum_{\mathrm{i}=1}^{\mathrm{n}}\left[\mathrm{d}_{\mathrm{ve}}\left(\mathrm{S}_{\mathrm{n}}, \mathrm{i}\right)+\mathrm{d}_{\mathrm{ve}}\left(\mathrm{C}_{\mathrm{n}-1}, \mathrm{i}\right)-\left(\begin{array}{c}
\mathrm{n}-1 \\
\mathrm{i}
\end{array}\right)\right] x^{\mathrm{i}} \\
& =\sum_{\mathrm{i}=1}^{\mathrm{n}} \mathrm{d}_{\mathrm{ve}}\left(\mathrm{S}_{\mathrm{n}}, \mathrm{i}\right) x^{\mathrm{i}} \quad+\sum_{\mathrm{i}=1}^{\mathrm{n}} \mathrm{d}_{\mathrm{ve}}\left(\mathrm{C}_{\mathrm{n}-1}, \mathrm{i}\right) x^{\mathrm{i}}-\sum_{\mathrm{i}=1}^{\mathrm{n}}\left(\begin{array}{c}
\mathrm{n}-1 \\
\mathrm{i}
\end{array}\right) x^{\mathrm{i}}
\end{aligned}
$$

W have,

$$
\begin{aligned}
& \mathrm{d}_{\mathrm{ve}}\left(\mathrm{C}_{\mathrm{n}-1}, \mathrm{i}\right)=0 \text { if } \mathrm{i}<\left\lceil\begin{array}{c}
\mathrm{n}-1 \\
4
\end{array}\right] \text { or } \mathrm{i}=\mathrm{n} \\
& \therefore \sum_{\mathrm{i}=1}^{\mathrm{n}} \mathrm{d}_{\mathrm{ve}}\left(\mathrm{C}_{\mathrm{n}-1}, \mathrm{i}\right) x^{\mathrm{i}}=\sum_{\mathrm{i}=\left[\frac{\mathrm{n}-1}{4}\right]}^{\mathrm{n}-1} \mathrm{~d}_{\mathrm{ve}}\left(\mathrm{C}_{\mathrm{n}-1}, \mathrm{i}\right) x^{\mathrm{i}} \\
& =\mathrm{D}_{\text {ve }}\left(\mathrm{C}_{\mathrm{n}-1}, x\right) \\
& \sum_{\mathrm{i}=1}^{\mathrm{n}} \mathrm{d}_{\mathrm{ve}}\left(\mathrm{S}_{\mathrm{n}}, \mathrm{i}\right) x^{\mathrm{i}}=\mathrm{D}_{\mathrm{ve}}\left(\mathrm{S}_{\mathrm{n}}, x\right) \\
& \sum_{\mathrm{i}=1}^{\mathrm{n}}\left(\begin{array}{c}
\mathrm{n}-1 \\
\mathrm{i}
\end{array}\right) x^{\mathrm{i}}=\left(\begin{array}{c}
\mathrm{n}-1 \\
1
\end{array}\right) x^{1}+\left(\begin{array}{c}
\mathrm{n}-2 \\
2
\end{array}\right) x^{2}+\ldots \ldots+\left(\begin{array}{c}
\mathrm{n}-1 \\
\mathrm{n}-1
\end{array}\right) x^{\mathrm{n}-1} \\
& =1+\left(\begin{array}{c}
\mathrm{n}-1 \\
1
\end{array}\right) x+\left(\begin{array}{c}
\mathrm{n}-1 \\
2
\end{array}\right) x^{2}+\ldots\left(\begin{array}{c}
\mathrm{n}-1 \\
\mathrm{n}-1
\end{array}\right) x^{\mathrm{n}-1}-1 \\
& =(1+x)^{\mathrm{n}-1}-1 \\
& \therefore \mathrm{D}_{\mathrm{ve}}\left(\mathrm{W}_{\mathrm{n}}, x\right)=\mathrm{D}_{\mathrm{ve}}\left(\mathrm{S}_{\mathrm{n}}, x\right)+\mathrm{D}_{\mathrm{ve}}\left(\mathrm{C}_{\mathrm{n}-1}, x\right)-\left((1+x)^{\mathrm{n}-1}-1\right) \\
& \text { (ii) } \mathrm{D}_{\mathrm{ve}}\left(\mathrm{W}_{\mathrm{n},}, x\right)=\sum_{\mathrm{i}=1}^{\mathrm{n}} \mathrm{d}_{\mathrm{ve}}\left(\mathrm{W}_{\mathrm{n}}, \mathrm{i}\right) x^{\mathrm{i}} \\
& =\sum_{\mathrm{i}=1}^{\mathrm{n}}\left[\mathrm{d}_{\mathrm{ve}}\left(\mathrm{W}_{\mathrm{n}-1}, \mathrm{i}-1\right)+\mathrm{d}_{\mathrm{ve}}\left(\mathrm{W}_{\mathrm{n}-2, \mathrm{i}} \mathrm{i}-1\right)+\mathrm{d}_{\mathrm{ve}}\left(\mathrm{W}_{\mathrm{n}-3, \mathrm{i}} \mathrm{i}-1\right)+\mathrm{d}_{\mathrm{ve}}\left(\mathrm{W}_{\mathrm{n}-4, \mathrm{i}-1)+}\right.\right. \\
& \left.\left(\begin{array}{c}
\mathrm{n}-5 \\
\mathrm{i}-1
\end{array}\right)\right] x^{\mathrm{i}}
\end{aligned}
$$

Since, $d_{v e}\left(W_{n}, 1\right)=0$ if $i>n$ or $i=0$

$$
\begin{aligned}
& \mathrm{D}_{\mathrm{ve}}\left(\mathrm{W}_{\mathrm{n}}, x\right)=\quad \sum_{\mathrm{i}=2}^{\mathrm{n}}\left[\mathrm{d}_{\mathrm{ve}}\left(\mathrm{W}_{\mathrm{n}-1}, \mathrm{i}-1\right)+\mathrm{d}_{\mathrm{ve}}\left(\mathrm{W}_{\mathrm{n}}-2, \mathrm{i}-1\right)+\mathrm{d}_{\mathrm{ve}}\left(\mathrm{W}_{\mathrm{n}}-3, \mathrm{i}-1\right)+\mathrm{d}_{\mathrm{ve}}\left(\mathrm{W}_{\mathrm{n}}-4, \mathrm{i}-1\right)\right] x^{\mathrm{i}} \\
& +\sum_{i=1}^{n}\left(\begin{array}{l}
n-5 \\
i-1
\end{array}\right) x^{i} \\
& =x \sum_{\mathrm{i}=2}^{\mathrm{n}} \mathrm{d}_{\mathrm{ve}}\left(\mathrm{W}_{\mathrm{n}-1}, \mathrm{i}-1\right) x^{\mathrm{i}-1}
\end{aligned}
$$

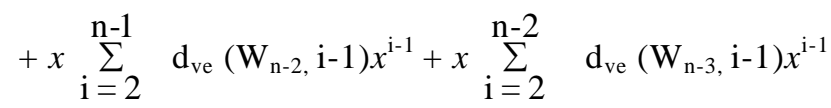




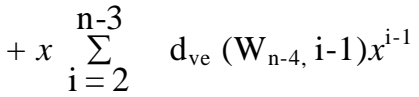

$$
\begin{aligned}
& +x \sum_{\mathrm{i}=1}^{\mathrm{n}-4}\left(\begin{array}{l}
\mathrm{n}-5 \\
\mathrm{i}-1
\end{array}\right) x^{\mathrm{i}-1} \\
& =x \mathrm{D}_{\mathrm{ve}}\left(\mathrm{W}_{\mathrm{n}-1}, x\right)+x \mathrm{D}_{\mathrm{ve}}\left(\mathrm{W}_{\mathrm{n}-2}, x\right)+x \mathrm{D}_{\mathrm{ve}}\left(\mathrm{W}_{\mathrm{n}-3}, x\right)+x \mathrm{D}_{\mathrm{ve}}\left(\mathrm{W}_{\mathrm{n}-4}, x\right)+x
\end{aligned}
$$

$$
\sum_{\mathrm{i}=1}^{\mathrm{n}-4}\left(\begin{array}{l}
\mathrm{n}-5 \\
\mathrm{i}-1
\end{array}\right) x^{\mathrm{i}-1}
$$

Consider

$$
\begin{aligned}
& \begin{aligned}
\sum_{\mathrm{i}=1}^{\mathrm{n}-4}\left(\begin{array}{c}
\mathrm{n}-5 \\
\mathrm{i}-1
\end{array}\right) x^{\mathrm{i}-1} & \\
= & \left(\begin{array}{c}
\mathrm{n}-5 \\
0
\end{array}\right) x^{\mathrm{o}}+\left(\begin{array}{c}
\mathrm{n}-5 \\
1
\end{array}\right) x^{1}+\left(\begin{array}{c}
\mathrm{n}-5 \\
2
\end{array}\right) x^{2}+\ldots+\left(\begin{array}{c}
\mathrm{n}-5 \\
\mathrm{n}-5
\end{array}\right) x^{\mathrm{n}-5} \\
& =1+\left(\begin{array}{c}
\mathrm{n}-5 \\
1
\end{array}\right) x^{1}+\left(\begin{array}{c}
\mathrm{n}-5 \\
2
\end{array}\right) x^{2}+\ldots+\left(\begin{array}{c}
\mathrm{n}-5 \\
\mathrm{n}-5
\end{array}\right) x^{\mathrm{n}-5} \\
& =(1+x)^{\mathrm{n}-5}
\end{aligned} \\
& \therefore \mathrm{D}_{\text {ve }}\left(\mathrm{W}_{\mathrm{n}}, x\right)=x \mathrm{D}_{\text {ve }}\left(\mathrm{W}_{\mathrm{n}-1}, x\right)+x \mathrm{D}_{\text {ve }}\left(\mathrm{W}_{\mathrm{n}-2}, x\right)+x \mathrm{D}_{\text {ve }}\left(\mathrm{W}_{\mathrm{n}-3}, x\right)+x \mathrm{D}_{\text {ve }}\left(\mathrm{W}_{\mathrm{n}-4}, x\right)+x(1+x)^{\mathrm{n}-5}
\end{aligned}
$$

\section{References}

[1] Alikhani. S and Peng. Y. H, Dominating sets and Domination polynomials of paths. Hindwi publishing corporation, International Journal Mathematics and Mathematical sciences 2009.

[2] Vijayan .A and Nagarajan .T, Vertex-edge dominating sets and vertex-edge domination polynomial of paths. International Journal of Mathematics Trends and Technology Volume 4, issue 11, Dec 2013.

[3] Vijayan .A and Nagarajan.T, Vertex-Edge dominating sets and vertex-edge domination polynomial of cycles submitted

[4] Sahib Shayyal Kahat, Abdul Jalil M. Khalaf, Roslan Hasni, Dominating sets and Domination polynomials of stars. ustralian Journal of Basic and applied Sciences, 8(6) : 383-386, 2014.

[5] Vijayan .A and Nagarajan .T Vertex-Edge Domination Polynomial of Graph. International Journal of Mathematical Archieve 5(2), (2014), 281-292.

[6] Bondy J.A. and Murthy U.S.R., Graph Theory with Applications, Elsevier Science Publishing Co, Sixth printing (1984).

[7] Haynes T.W., Hedetniemi S.T. and Slater P.J. Fundamentals of Domination in Graphs, Marcel Dekker, Newyork (1998). 